J. Math. Soc. Japan

Vol. 43, No. 2, 1991

\title{
Fourier transforms for affine automorphism groups on Siegel domains
}

\author{
By Junko INOUE
}

(Received Feb. 22, 1990)

(Revised May 23, 1990)

\section{Introduction.}

Let $G$ be a connected Lie group, $d g$ a left Haar measure on $G$ and $\pi$ be an irreducible unitary representation of $G$ on a Hilbert space $\mathcal{H}$. Then for an integrable function $\varphi$ on $G$ the Fourier transform with respect to $\pi$ is defined as the integrated operator $\pi(\varphi)=\int_{G} \pi(g) \varphi(g) d g$. As is well known, if $G$ is semisimple or nilpotent, then $\pi(\varphi)$ is a compact operator on $\mathscr{H}$ for any irreducible representation $\pi$ and any integrable function $\varphi$. But otherwise, $\pi(\varphi)$ is not always compact, thus characterization of $\varphi \in L^{1}(G)$ such that $\pi(\varphi)$ is compact is an important problem in representation theory for solvable Lie groups.

In [7], Khalil determined such functions for the $a x+b$ group by the "mean value over the subgroup of translations" (Example 3.1). In this paper we generalize this result to transitive groups of affine automorphisms on Siegel domains. More precisely, we treat connected and simply connected Lie groups $G$ whose Lie algebras $g$ are normal $j$-algebras (Definition 1.1) and their square integrable representations.

Our characterization is, roughly speaking, based on conditions of zero-sets of partial Euclidean Fourier transform on the abelian normal subgroup $G_{1}=\operatorname{exp~}_{1}$ (under the notations of 1.5). Identifying $G$ with $g_{1} \times\left(G_{1} \backslash G\right)$, we take the Euclidean Fourier transform $\mathscr{F}_{1} \varphi$ of $\varphi \leqq L^{1}(G)$ on $g_{1}$-part, which is a function on $g_{1}^{*} \times\left(G_{1} \backslash G\right)$. On the other hand, the unitary dual $\hat{G}$ of $G$ being parametrized by coadjoint orbits of $G$ on $\mathrm{g}^{*}$, square integrable representations correspond to open orbits, whose union is dense in $g^{*}$. For such a representation $\pi$ of $G$, let $\Omega$ be the corresponding open orbit, $\partial \Omega$ be its boundary in $g^{*}$. Considering the natural projection $p: g^{*} \rightarrow g_{1}^{*}$ defined by $p(l)=l l_{g_{1}}$ (restriction of $l$ to $g_{1}$ ), we show that $\pi(\varphi)$ is compact if and only if $\mathscr{I}_{1} \varphi$ vanishes on $p(\partial \Omega) \times\left(G_{1} \backslash G\right)$ (Theorem 2.2).

In section 1, we summarize preliminary results on structures of normal $j$-algebras and unitary representations of their corresponding groups. Our 
criterion for compactness is proved in section 2 , and at the same time we verify that $\pi(\varphi)$ is compact if and only if $\pi_{l}(\varphi)=0$ for all $l \in \partial \Omega$, where $\pi_{l}$ is the representation of $G$ corresponding to the orbit $G \cdot l$. Finally we give examples in section 3 .

\section{Preliminaries.}

DEFINITION 1.1. A triple $\left(\mathrm{g}, j, f_{0}\right)$ is a normal $j$-algebra if

(a) $g$ is a real completely solvable Lie algebra (i.e., $g$ admits a decreasing series of ideals $g_{i}$ such that $\operatorname{dim} g_{i} / g_{i+1}=1$, ,

(b) $j: \mathfrak{g} \rightarrow \mathfrak{g}$ is a complex structure (i.e., $j^{2}=-\mathbf{1}$ ),

(c) $\mathrm{g}^{-}=\{Y+\sqrt{-1} j Y ; Y \in \mathrm{g}\}$ is a Lie subalgebra of $\mathrm{g}^{c}$,

(d) $f_{0} \in \mathrm{g}^{*}$ has the properties

(1) $f_{0}([Y, j Y])>0$ for all $Y \in g-\{0\}$,

(2) $f_{0}\left(\left[\mathfrak{g}^{-}, \mathfrak{g}^{-}\right]\right)=\{0\}$.

EXAMPLE $1.2(a x+b$ algebra). Let $g$ be the Lie algebra of the $a x+b$ group $G=\left\{\left(\begin{array}{ll}a & b \\ 0 & 1\end{array}\right) ; a, b \in \boldsymbol{R}, a>0\right\}$, that is, $\mathfrak{g}=\boldsymbol{R} X+\boldsymbol{R} Y$, where $X=\left(\begin{array}{ll}1 & 0 \\ 0 & 0\end{array}\right), Y=\left(\begin{array}{ll}0 & 1 \\ 0 & 0\end{array}\right)$. Define a linear map $j$ on $\mathrm{g}$ by $j X=-Y, j Y=X$, and a linear form $f_{0}$ by $f_{0}(X)=0, f_{0}(Y)=-1$. Then the triple $\left(g, j, f_{0}\right)$ is a normal $j$-algebra.

EXAMPLE 1.3. Let $\mathrm{g}=\boldsymbol{R}$-span $\left\{X_{1}, X_{2}, W, Z, Y_{1}, Y_{2}\right\}$, where non-trivial bracket relations are;

$$
\begin{array}{lll}
{\left[X_{1}, W\right]=-\frac{1}{2} W,} & {\left[X_{1}, Z\right]=\frac{1}{2} Z,} & {\left[X_{1}, Y_{1}\right]=Y_{1},} \\
{\left[X_{2}, W\right]=\frac{1}{2} W,} & {\left[X_{2}, Z\right]=\frac{1}{2} Z,} & {\left[X_{2}, Y_{2}\right]=Y_{2},} \\
{[W, Z]=Y_{2},} & {\left[W, Y_{1}\right]=Z .} &
\end{array}
$$

Define a linear map $j$ by $j X_{1}=-Y_{1}, j X_{2}=-Y_{2}, j W=-Z, j Z=W, j Y_{1}=X_{1}$, $j Y_{2}=X_{2}$, and a linear form $f_{0}$ by $f_{0}\left(Y_{1}\right)=f_{0}\left(Y_{2}\right)=-1, f_{0}(Z)=f_{0}\left(X_{1}\right)=f_{0}\left(X_{2}\right)=$ $f_{0}(W)=0$. Then $\left(g, j, f_{0}\right)$ is a normal $j$-algebra. $g$ can be realized as a subalgebra of $4 \times 4$ real matrix algebra (with ordinary bracket operation) by

$$
x_{1} X_{1}+x_{2} X_{2}+w W+z Z+y_{1} Y_{1}+y_{2} Y_{2}=\left(\begin{array}{cccc}
x_{2} & w & 0 & y_{2} \\
0 & \left(x_{1}+x_{2}\right) / 2 & w & z \\
0 & 0 & x_{1} & y_{1} \\
0 & 0 & 0 & 0
\end{array}\right)
$$

for $x_{1}, x_{2}, w, z, y_{1}, y_{2} \in R$.

REMARK 1.4. Regarding a normal $j$-algebra $\left(g, j, f_{0}\right)$, it is known that the group $G=\operatorname{expg}$ can be realized as an affine automorphism group acting simply 
and transitively on a Siegel domain of type II, and vice versa. (In Example 1.3, the corresponding Siegel domain is the Siegel upper-half plane of degree 2.) For details concerning homogeneous Siegel domains, we refer the reader to [6], [10], for example.

1.5. Here we summarize the fundamental structure of a normal $j$-algebra (see [10, Theorem 2, Chapter 2] or [11, Theorem 5.13]). Let $\left(g, j, f_{0}\right)$ be a normal $j$-algebra. Let $\Lambda$ be the symmetric positive definite bilinear form $\Lambda(X, Y)=$ $f_{0}([X, j Y])$ on $\mathfrak{g}$, and let $\mathfrak{a}$ be the orthogonal complement of $\mathfrak{h}=[\mathfrak{g}, \mathfrak{g}]$ with respect to $A$. Then $\mathfrak{a}$ is an abelian subalgebra of $\mathfrak{g}, \mathfrak{g}=\mathfrak{a}+\mathfrak{h}$, and the adjoint representation of $\mathfrak{a}$ on $\mathfrak{h}$ is real diagonalizable. Thus we have a decomposition of $\mathfrak{h}$ into root spaces,

$$
\mathfrak{h}=\sum_{\alpha \in \mathfrak{a} *} \mathfrak{g}^{\alpha},
$$

where $\mathfrak{g}^{\alpha}=\{X \in \mathfrak{h} ;[A, X]=\alpha(A) X$ for all $A \in \mathfrak{a}\}$, and only finitely many $\mathfrak{g}^{\alpha}$ 's can be non-zero.

Let $\left\{\mathfrak{g}^{\alpha}{ }^{k}\right\}, 1 \leqq k \leqq r$ be those root spaces for which $j\left(\mathrm{~g}^{\alpha}\right) \subset \mathfrak{a}$. Then $\operatorname{dim} \mathrm{g}^{\alpha_{k}}=1$ and $r=\operatorname{dim} \mathfrak{a}$ ( $r$ is called the rank of $\mathfrak{g}$ ), and we can order $\alpha_{1}, \cdots, \alpha_{r}$ in an appropriate way so that all the other roots are of the form

$$
\begin{aligned}
& \left(\alpha_{m}+\alpha_{k}\right) / 2, \quad\left(\alpha_{m}-\alpha_{k}\right) / 2 \quad 1 \leqq k<m \leqq r, \\
& \alpha_{k} / 2 \quad 1 \leqq k \leqq r
\end{aligned}
$$

(not all the possibilities need occur). Let

$$
\begin{aligned}
& \mathrm{g}_{0}=\mathfrak{a}+\sum_{1 \leqq k<m \leqq r} \mathrm{~g}^{\left(\alpha_{m}-\alpha_{k}\right) / 2}, \\
& \mathrm{~g}_{1 / 2}=\sum_{1 \leqq k \leqq r} \mathrm{~g}^{\alpha_{k} / 2}, \\
& \mathrm{~g}_{1}=\sum_{1 \leqq k \leqq r} \mathrm{~g}^{\alpha_{k}}+\sum_{1 \leqq k<m \leqq r} \mathrm{~g}^{\left(\alpha_{m}+\alpha_{k}\right) / 2} .
\end{aligned}
$$

Then

$$
\left[\mathfrak{g}_{\nu}, \mathfrak{g}_{\mu}\right] \subset \mathfrak{g}_{\nu+\mu}
$$

(with the convention that if $\nu+\mu \neq 0,1 / 2$, nor 1 , then $g_{\nu+\mu}=\{0\}$ ), and $j\left(g_{1 / 2}\right)=$ $\mathfrak{g}_{1 / 2}, j\left(\mathfrak{g}_{0}\right)=\mathfrak{g}_{1}$. More specifically,

$$
\begin{aligned}
& j\left(\mathrm{~g}^{\left(\alpha_{m}+\alpha_{k}\right) / 2}\right)=\mathrm{g}^{\left(\alpha_{m}-\alpha_{k}\right) / 2} \quad 1 \leqq k<m \leqq r, \\
& j\left(\mathrm{~g}^{\alpha_{k / 2}}\right)=\mathrm{g}^{\alpha_{k / 2}} \quad 1 \leqq k \leqq r .
\end{aligned}
$$

Let $U_{k}$ be a non-zero element of $\mathrm{g}^{\alpha_{k}}$ such that $\left[j U_{k}, U_{k}\right]=U_{k}$, then

$$
\alpha_{l}\left(j U_{k}\right)=\delta_{l, k} \text { (Kronecker's delta) } 1 \leqq k, l \leqq r .
$$

1.6. We next consider unitary representations of $G=\exp g$. Since $G$ is exponential (i.e., the exponential map from $g$ to $G$ is a diffeomorphism), the unitary dual $\hat{G}$ of $G$ is parametrized by orbits of the coadjoint action of $G$ on 
the dual space $g^{*}$ of $g$. Here we give an outline of the parametrization of $\hat{G}$ for an exponential group $G$. To a coadjoint orbit $\Omega$, a class $\Theta(\Omega) \in \hat{G}$ corresponds in the following way: Let $f \in \Omega$, then there exists a subalgebra $\mathfrak{b}=\mathfrak{b}_{f}$ satisfying the following conditions.

(1) $f([\mathfrak{b}, \mathfrak{b}])=\{0\}$.

(2) $\mathfrak{b}$ has the maximal dimension among all subalgebras satisfying (1). (Such $\mathfrak{b}$ is called a real polarization at $f$.)

(3) (The Pukanszky condition) The affine space $\mathfrak{b}^{\perp}+f$, where $\mathfrak{b}^{\perp}=\left\{l \in \mathfrak{g}^{*}\right.$; $\left.l l_{\mathfrak{b}}=0\right\}$, is contained in $\Omega$.

Let $\pi=\pi(f, \mathfrak{b})$ be the representation induced by the character $\chi_{f}(\exp X)=$ $e^{\sqrt{-1} f(X)}$ of $B=\exp \mathfrak{b}$. Then $\pi$ is irreducible and its equivalence class is independent of $f \in \Omega$ and $\mathfrak{b}$. Thus, $f \rightarrow \pi(f, \mathfrak{b})$ gives a map $\Theta$ from coadjoint orbits $\mathrm{g}^{*} / G$ to $\hat{G}$. $\Theta$ is bijective and called the Kirillov-Bernat map [2].

We now return to a normal $j$-algebra $\left(g, j, f_{0}\right)$ of rank $r$ and $G=\operatorname{expg}$. Then $G$ has open coadjoint orbits, whose union is dense in $g^{*}$. They correspond to the classes of square integrable representations of $G$ [4]. The following proposition describes these open orbits. Retaining the notations in 1.5 , we note that the subgroup $G_{0}=\exp g_{0}$ acts on $g_{1}^{*}$ by the coadjoint action since $g_{1}$ is an ideal in $\mathrm{g}$.

Proposition 1.7 [8, Proposition 1.4], [12, Proposition 3.3.1].

(1) $G_{0}$ has open orbits in $\mathrm{g}_{1}^{*}$, and the union of open orbits is dense. More precisely, noting the direct sum decomposition $\boldsymbol{g}_{1}=\sum_{1 \leqq i \leqq r} \boldsymbol{R} U_{i} \oplus_{1 \leqq k<m \leqq r} \boldsymbol{g}^{\left(\alpha_{m}+\alpha_{k}\right) / 2}$, define $U_{i}^{*} \in g_{1}^{*}$ by $U_{i}^{*}\left(U_{k}\right)=\delta_{i, k}$ and $U_{i}^{*} \mid g_{\left(\alpha_{m}+\alpha_{k}\right) / 2}=0$, for each $i(1 \leqq i \leqq r)$. Then

$$
f_{\varepsilon}=\sum_{1 \leqq i \leqq r} \varepsilon_{i} U_{i}^{*}, \quad \varepsilon \in I=\left\{\left(\varepsilon_{1}, \cdots, \varepsilon_{r}\right) ; \varepsilon_{i}= \pm 1\right\}
$$

form a system of representatives of open orbits of $G_{0}$ in $g_{1}^{*}$.

(2) According to the direct sum decomposition $\mathrm{g}=\mathrm{g}_{0} \oplus \mathrm{g}_{1 / 2} \oplus \mathrm{g}_{1}$, let us regard $\mathrm{g}^{*}=\mathrm{g}_{0}^{*} \oplus \mathrm{g}_{1 / 2}^{*} \oplus \mathrm{g}_{1}^{*}$. Then the open coadjoint orbits in $\mathrm{g}^{*}$ are

$$
G \cdot f_{\varepsilon}=g_{0}^{*}+g_{1 / 2}^{*}+G_{0} \cdot f_{\varepsilon}, \quad \varepsilon \in I .
$$

1.8. Lastly we introduce the construction of real polarization due to $\mathrm{M}$. Vergne [2], to be used later. Let $g$ be a completely solvable Lie algebra, and $\left(\mathfrak{g}_{i}\right)_{0 \leqq i \leqq n=\operatorname{dim} g}$ a flag of ideals (i.e., $\left(\mathfrak{g}_{i}\right)$ is an increasing sequence of ideals in $\mathfrak{g}$ such that $\left.\operatorname{dim} \mathfrak{g}_{i}=i\right)$. Then we get a real polarization $\mathfrak{b}$ at $f \in \mathrm{g}^{*}$ in the following way: Denote by $\lambda_{f}$ the alternative bilinear form $\lambda_{f}(X, Y)=f([X, Y])$ on $\mathrm{g}$, and by $\lambda_{i}$ the restriction of $\lambda_{f}$ to $g_{i} \times g_{i}$. Let $g\left(\lambda_{i}\right)=\left\{X \in g_{i} ; \lambda_{i}(X, Y)=0\right.$ for all $\left.Y \in \mathfrak{g}_{i}\right\}$, the radical of $\lambda_{i}$. Then, $\mathfrak{b}=\sum_{i=1}^{n} \mathfrak{g}\left(\lambda_{i}\right)$ is a real polarization at $f$ satisfying the Pukanszky condition. 


\section{A characterization of functions transformed into compact operators.}

Let $g$ be a normal $j$-algebra of rank $r, G=\exp g, d g$ a left Haar measure on $G$, and $\pi$ be an irreducible unitary representation of $G$ corresponding to an open orbit $\Omega$. We retain the notations of Theorem 1.5. In this section, we will characterize $L^{1}$-functions whose Fourier transforms with respect to $\pi$ are compact operators.

Definition 2.1. Let $\varphi \in L^{1}(G)$ and $d X$ be a Lebesgue measure on $g_{1}$. We define the partial Euclidean Fourier transform $\mathscr{F}_{1} \varphi$ on $G_{1}=\exp g_{1}$ by

$$
\mathscr{F}_{1} \varphi(l)(g)=\int_{\mathfrak{g}_{1}} e^{\sqrt{-1}\langle l, X\rangle} \varphi((\exp X) g) d X,
$$

for $l \in \mathrm{g}_{1}^{*}$ and (almost everywhere) $g \boxminus G$.

Since $\mathscr{F}_{1} \varphi(l)\left(g_{1} g\right)=e^{-\sqrt{-1}\langle l, Y\rangle} \mathscr{F}_{1} \varphi(l)(g)$ for all $g_{1}=\exp Y \in G_{1},\left|\mathscr{F}_{1} \varphi(l)(g) \Delta_{G}(g)\right| \in$ $L^{1}\left(G_{1} \backslash G, d_{r} \dot{g}\right)$ for a fixed $l$, where $d_{r} \dot{g}$ is a right Haar measure on $G_{1} \backslash G$ and $\Delta_{G}$ is the modular function of $G$.

We will prove the following theorem.

TheOREM 2.2. Let $\mathrm{g}$ be a normal j-algebra of rank $r, G=\exp \mathfrak{g}, d g$ a left Haar measure on $G,\left(\pi, \mathcal{H}_{\pi}\right)$ an irreducible representation of $G$ corresponding to an open orbit $\Omega$ in $\mathrm{g}^{*}$ with usual topology. Denoting the closure of $\Omega$ by $\operatorname{cl}(\Omega)$, let $\partial \Omega=\operatorname{cl}(\Omega) \backslash \Omega$. And we write $\pi_{l}$ for the irreducible representation of $G$ corresponding to an orbit $G \cdot l$, where $l \in \mathrm{g}^{*}$. Then for $\varphi \in L^{1}(G, d g)$, the following claims are equivalent.

1. $\pi(\varphi)$ is a compact operator on $\mathscr{H}_{\pi}$.

2. $\mathscr{I}_{1} \varphi\left(l_{1}\right)(g)=0$ for all $l_{1}=\left.l\right|_{g_{1}}$ such that $l \in \partial \Omega$.

3. $\pi_{l}(\varphi)=0$ for all $l \in \partial \Omega$.

REMARK 2.3. From Arsac's result [1], $\pi(\varphi)$ is compact if and only if $\sigma(\varphi)=0$ for all $\sigma \in(\overline{\{\pi\}} \backslash\{\pi\})$, where $\overline{\{\pi\}}$ denotes the closure of $\{\pi\}$ in $\hat{G}$ relative to the Fell topology (i.e., $\sigma \in \overline{\{\pi\}}$ if and only if $\sigma$ is weakly contained in $\{\pi\}$ ). On the other side if $G$ is an exponential group, the Kirillov-Bernat map $\Theta: \mathrm{g}^{*} / G \rightarrow \hat{G}$ is known to be bijective and continuous with quotient topology on $\mathfrak{g}^{*} / G$ and the Fell topology on $\hat{G}$ (see [9]). Thus claim 1 implies claim 3 . But it has not been known whether $\Theta^{-1}$ is continuous or not in general.

ProOF OF THE THEOREM. We will prove in Step 1 that claim 2 and claim 3 are equivalent, and in Step 2 that claim 2 implies claim 1.

Step 1. We first suppose claim 2. Take a flag of ideals by refining the series $0 \subset g_{1} \subset g$, and construct a polarization $\mathfrak{b}_{l}$ at $l \in \partial \Omega$ as in 1.8. Let $\pi_{l}$ be regarded as induced from the character $\chi_{l}(\exp X)=e^{\sqrt{-1}\langle l, X\rangle}$ of $B_{l}=\exp _{l} . \quad \pi_{l}$ is modeled in a space of functions $\zeta$ on $G$ such that $\zeta(b g)=\chi_{l}(b)\left(\Delta_{B_{l}}(b) / \Delta_{G}(b)\right)^{1 / 2} \zeta(g)$ 
for all $b \in B_{l}$ and $g \in G$, with right translation ; $\left(\pi_{l}(g) \zeta\right)(x)=\zeta(x g)$. Since $g_{1}$ is an abelian ideal, we have $\mathfrak{g}_{1} \subset \mathfrak{b}_{l}$, so that for $g_{1} \in G_{1}, x \in G$,

$$
\left(\pi_{l}\left(g_{1}\right) \zeta\right)(x)=\chi_{l}\left(x g_{1} x^{-1}\right) \zeta(x)=\chi_{x-1 \cdot l}\left(g_{1}\right) \zeta(x) .
$$

Let us identify $G$ with $G_{1} \times\left(G_{1} \backslash G\right)$ by taking a global section $s$ of $G_{1} \backslash G$, and choose the right Haar measure $d \dot{g}$ on $G_{1} \backslash G$ so that $\Delta_{G}^{-1}(g) d g=d X d \dot{g}$ for $g=(\exp X) s(\dot{g})$ with $X \in g_{1}, \dot{g} \in\left(G_{1} \backslash G\right)$. Then

$$
\begin{aligned}
& \left(\pi_{l}(\varphi) \zeta\right)(x)=\int_{G}\left(\pi_{l}(g) \zeta\right)(x) \varphi(g) d g \\
= & \int_{g_{1}} \int_{G_{1} \backslash G} \chi_{x-1 \cdot l}(\exp X)\left(\pi_{l}(s(\dot{g})) \zeta\right)(x) \varphi((\exp X) s(\dot{g})) \Delta_{G}(s(\dot{g})) d X d \dot{g} \\
= & \int_{G_{1} \backslash G} \mathscr{F}_{1} \varphi\left(x^{-1} \cdot l_{1}\right)(s(\dot{g}))\left(\pi_{l}(s(\dot{g})) \zeta\right)(x) \Delta_{G}(s(\dot{g})) d \dot{g}
\end{aligned}
$$

$\left(l_{1}=\left.l\right|_{g_{1}}\right)$. The integrand is 0 from the assumption, which implies claim 3.

We next prove that claim 3 implies claim 2 . For $l_{1}=\left.l\right|_{g_{1}}, l \in \partial \Omega$, consider the induced representation of $G$ from the character $\chi_{l}(\exp X)=e^{\sqrt{-1}\langle l, X\rangle}$ of $G_{1}$; $\tau=\operatorname{ind}_{G_{1}}^{G} \chi_{l}$. It is known that $\tau$ decomposes into the direct integral of irreducible representations of $G$ corresponding to coadjoint orbits which intersect the affine space $\mathrm{g}_{1}^{\frac{1}{1}}+l$ (e.g., [3]). Now, recall that $\Omega$ has the following description;

$$
\Omega=G \cdot f=G_{0} \cdot f_{1}+\mathrm{g}_{1 / 2}^{*}+\mathrm{g}_{0}^{*},
$$

where $f_{1}=\left.f\right|_{g_{1}}$ (Proposition 1.7). Thus $\Omega+g_{1}^{1}=\Omega$, and $\partial \Omega+g_{1}^{1}=\partial \Omega$, which implies that the representations appearing in the decomposition of $\tau$ correspond to orbits included in $\partial \Omega$. Thus claim 3 implies $\tau(\varphi)=0$, that is, realizing $\tau$ in $L^{2}\left(G_{1} \backslash G\right)$ with right translation,

$$
\begin{aligned}
& (\tau(\varphi) v)(\dot{x}) \\
= & \int_{g_{1}} \int_{G_{1} \backslash G} \chi_{l}\left(\dot{x}(\exp X) \dot{x}^{-1}\right)(\tau(s(\dot{g})) v)(\dot{x}) \varphi((\exp X) s(\dot{g})) \Delta_{G}(s(\dot{g})) d X d \dot{g} \\
= & \int_{G_{1} \backslash G} \mathscr{F}_{1} \varphi\left(\dot{x}^{-1} \cdot l_{1}\right)(s(\dot{g}))(\tau(s(\dot{g})) v)(\dot{x}) \Delta_{G}(s(\dot{g})) d \dot{g} \\
= & 0
\end{aligned}
$$

for all $v \in L^{2}\left(G_{1} \backslash G\right)$ and almost all $\dot{x} \in G_{1} \backslash G$. And then for $v \in C_{c}^{\infty}\left(G_{1} \backslash G\right)$, we get $(\tau(\varphi) v)(\dot{e})=0$ ( $\dot{e}$ is the unit element of $\left.G_{1} \backslash G\right)$ since $\tau(\varphi) v$ is a continuous function. Thus

$$
(\tau(\varphi) v)(\dot{e})=\int_{G_{1} \backslash G} \mathscr{F}_{1} \varphi\left(l_{1}\right)(s(\dot{g})) v(s(\dot{g})) \Delta_{G}(s(\dot{g})) d \dot{g}=0
$$

for all $v \in C_{c}^{\infty}\left(G_{1} \backslash G\right)$. It follows that $\mathscr{F}_{1} \varphi\left(l_{1}\right)(s(\dot{g}))=0$ for almost all $\dot{g}$ and claim 2 is verified. 
Step 2. Let $\mathfrak{n}=\mathfrak{g}_{1}+\mathfrak{g}_{1 / 2}$ and $N=\exp \mathfrak{n}$, which is a normal subgroup of $G$, and we have $G=N G_{0}$. As in Step 1 , construct a real polarization $\mathfrak{b}_{f}$ at $f \in \Omega$ taking a flag of ideals which refines the series $0 \subset g_{1} \subset g$ (see 1.8). Then $\mathfrak{g}_{1} \subset \mathfrak{b}_{f} \subset \mathfrak{n}$. In fact, the map $F: \mathfrak{g}_{0} \rightarrow \mathfrak{g}_{1}^{*} ; F(X)=f_{1}([\cdot, X])$, where $f_{1}=\left.f\right|_{\mathfrak{g}_{1}}$, is a linear isomorphism since $G_{0} \cdot f_{1}$ is open in $g_{1}^{*}$ (Proposition 1.7) and $F$ is the differential of the map $\tilde{F}: G_{0} \rightarrow g_{1}^{*} ; \tilde{F}\left(g_{0}\right)=g_{0} \cdot f_{1}$, at $e$ (the unit element). Noting that $\mathfrak{g}_{1}$ is central in $\mathfrak{n}$, we can thus easily see that for any ideal $\mathfrak{g}^{\prime}$ of $\mathfrak{g}$ including $g_{1}$, the radical of the restriction of the bilinear form $\lambda_{f}=f([\cdot, \cdot])$ to $\mathfrak{g}^{\prime} \times \mathfrak{g}^{\prime}$ is included in $\mathfrak{n}$, so that $\mathfrak{b}_{f} \subset \mathfrak{n}$.

Thus we regard $\pi=\operatorname{ind}_{B f}^{G} \chi_{f}$ as induced from the irreducible representation

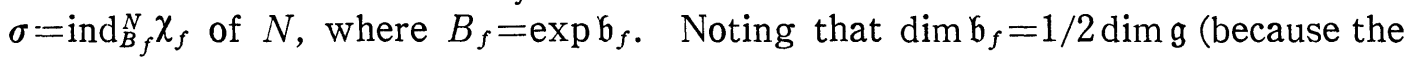
bilinear form $\lambda_{f}$ is non-singular), let $\left\{X_{1}, \cdots, X_{m}, Y_{1}, \cdots, Y_{2 k}\right\}$ be a basis for $\mathfrak{n}$ such that $\mathfrak{g}_{1}=\boldsymbol{R}$-span $\left\{X_{1}, \cdots, X_{m}\right\}, \mathfrak{b}_{f}=\boldsymbol{R}$-span $\left\{\mathfrak{g}_{1}, Y_{1}, \cdots, Y_{k}\right\}$, and each $\mathfrak{n}_{i}=\boldsymbol{R}$-span $\left\{\mathfrak{g}_{1}, Y_{1}, \cdots, Y_{i}\right\}$ is a subalgebra with $\mathfrak{n}_{i}$ an ideal in $\mathfrak{n}_{i+1}$. (Such basis is called a weak Malcev basis.) Through the diffeomorphism $\Phi: \mathfrak{g}_{1}+\boldsymbol{R}^{2 k} \rightarrow N$ defined by $\left(X, y_{1}, \cdots, y_{2 k}\right) \rightarrow \exp X \exp y_{1} Y_{1} \cdots \exp y_{2 k} Y_{2 k} \in N$, we transfer Euclidean measures $d X, d X d y_{1} \cdots d y_{k}$ and $d X d y_{1} \cdots d y_{2 k}$ to Haar measures $d g_{1}$ on $G_{1}$, $d b$ on $B_{f}$ and $d n$ on $N$ respectively, and $d y_{k+1} \cdots d y_{2 k}$ to an $N$-invariant measure $d \dot{n}$ on $B_{f} \backslash N$. In the sequel, we realize $\sigma$ in $\mathcal{H}_{\sigma}=L^{2}\left(\boldsymbol{R}^{k}, d y_{k+1} \cdots d y_{2 k}\right)$ and using the right Haar measure $d g_{0}$ on $G_{0}$ such that $\Delta_{G}^{-1}(g) d g=d n d g_{0}$, realize $\pi$ in the space $L^{2}\left(G_{0}, \mathscr{H}_{\sigma}, d g_{0}\right)$ of $\mathscr{T}_{\sigma}$-valued $L^{2}$-function on $G_{0}$ :

$$
\left(\pi\left(n g_{0}\right) \xi\right)\left(x_{0}\right)=\sigma\left(x_{0} n x_{0}^{-1}\right) \xi\left(x_{0} g_{0}\right)=\sigma^{x_{0}}(n) \xi\left(x_{0} g_{0}\right)
$$

for $\xi \in L^{2}\left(G_{0}, \mathscr{H}_{\sigma}\right), n g_{0} \in G=N G_{0}$. ( $\sigma^{x_{0}}$ denotes the representation of $N$ in $\mathscr{H}_{\sigma}$ given by $n \rightarrow \sigma\left(x_{0} n x_{0}^{-1}\right)$.) Since the normal subgroup $G_{1}$ is central in $N$, we have

$$
\left(\pi\left(g_{1}\right) \xi\right)\left(x_{0}\right)=\chi_{f}\left(x_{0} g_{1} x_{0}^{-1}\right) \xi\left(x_{0}\right)=\chi_{x_{0}^{-1} \cdot f}\left(g_{1}\right) \xi\left(x_{0}\right)
$$

for $g_{1} \in G_{1}, x_{0} \in G_{0}$. Writing $\boldsymbol{y}=\left(y_{1}, \cdots, y_{2 k}\right) \in \boldsymbol{R}^{2 k}, \Psi(\boldsymbol{y})=\Phi(0, \boldsymbol{y}), d \boldsymbol{y}=d y_{1} \cdots d y_{2 k}$, for convenience' sake, we get

$$
\begin{aligned}
& (\pi(\varphi) \xi)\left(x_{0}\right)=\int_{N} \int_{G_{0}}\left(\pi\left(n g_{0}\right) \xi\right)\left(x_{0}\right) \varphi\left(n g_{0}\right) \Delta_{G}\left(n g_{0}\right) d n d g_{0} \\
= & \int_{\theta_{1}} \int_{R^{2} k} \int_{G_{0}} \chi_{x_{0}^{-1} \cdot f}(\exp X)\left(\pi\left(\Psi(\boldsymbol{y}) g_{0}\right) \xi\right)\left(x_{0}\right) \varphi\left((\exp X) \Psi(\boldsymbol{y}) g_{0}\right) \Delta_{G}\left(g_{0}\right) d X d \boldsymbol{y} d g_{0} \\
= & \int_{\boldsymbol{R}^{2 k}} \int_{G_{0}} \mathscr{F}_{1} \varphi\left(x_{0}^{-1} \cdot f_{1}\right)\left(\Psi(\boldsymbol{y}) g_{0}\right) \sigma^{x_{0}}(\Psi(\boldsymbol{y})) \xi\left(x_{0} g_{0}\right) \Delta_{G}\left(g_{0}\right) d \boldsymbol{y} d g_{0} .
\end{aligned}
$$

Here we need a lemma.

LEMMA 2.4. Let $\beta$ be a function on $G$ such that $\beta=\beta_{N} \cdot \beta_{0}$, where $\beta_{N} \in C_{c}(N), \quad \beta_{0} \in C_{c}\left(G_{0}\right)$ (compactly supported continuous functions), and let $\kappa \in C_{c}\left(G_{0}\right)$. Then $\kappa \cdot \pi(\beta)$ is a compact operator on $L^{2}\left(G_{0}, \mathcal{H}_{\sigma}\right)$. 
PROOF. Noting that $\mathscr{H}_{\sigma}=L^{2}\left(B_{f} \backslash N\right)$, we write $\boldsymbol{\xi}=\boldsymbol{\xi}\left(g_{0}\right)=\boldsymbol{\xi}\left(g_{0}\right)_{(\dot{n})}$ for $\xi \in L^{2}\left(G_{0}, \mathscr{H}_{\sigma}\right)$ with variables $g_{0} \in G_{0}, \dot{n} \in B_{f} \backslash N$. For a fixed $x_{0} \in G_{0}$, define an operator $R\left(x_{0}\right): L^{2}\left(G_{0}, \mathscr{H}_{\sigma}\right) \rightarrow \mathscr{H}_{\sigma}$ by

$$
R\left(x_{0}\right) \xi=\int_{G_{0}} \xi\left(x_{0} g_{0}\right) \beta_{0}\left(g_{0}\right) \Delta_{G}\left(g_{0}\right) d g_{0} .
$$

$R\left(x_{0}\right)$ is well-defined as a bounded operator because

And we get

$$
\begin{aligned}
\left\|R\left(x_{0}\right) \xi\right\|_{\mathscr{H}_{\sigma}}^{2} & =\int_{B_{f} \backslash N}\left|\int_{G_{0}} \xi\left(x_{0} g_{0}\right)_{(\dot{n})} \beta_{0}\left(g_{0}\right) \Delta_{G}\left(g_{0}\right) d g_{0}\right|^{2} d \dot{n} \\
& \leqq \int_{B_{f} \backslash N} \int_{G_{0}}\left|\xi\left(x_{0} g_{0}\right)_{(\dot{n})}\right|^{2} d g_{0}\left\|\beta_{0} \cdot \Delta_{G}\right\|_{L^{2}\left(G_{0}\right)}^{2} d \dot{n} \\
& =\Delta_{G_{0}}\left(x_{0}\right)\|\xi\|_{L^{2}\left(G_{0}, \mathscr{H}_{\sigma}\right)}^{2}\left\|\boldsymbol{\beta}_{0} \cdot \Delta_{G}\right\|_{L^{2}\left(G_{0}\right)}^{2} .
\end{aligned}
$$

$$
\begin{aligned}
(\pi(\beta) \xi)\left(x_{0}\right) & =\int_{N} \int_{G_{0}} \sigma^{x_{0}}(n) \xi\left(x_{0} g_{0}\right) \beta_{N}(n) \beta_{0}\left(g_{0}\right) \Delta_{G}\left(g_{0}\right) d n d g_{0} \\
& =\int_{N} \sigma^{x_{0}}(n) R\left(x_{0}\right) \xi \beta_{N}(n) d n=\sigma^{x_{0}}\left(\beta_{N}\right) R\left(x_{0}\right) \xi
\end{aligned}
$$

Now let $\left\{\xi_{\iota} ; \iota \in I\right\}$ be a sequence which converges weakly to 0 in $L^{2}\left(G_{0}, \mathscr{H}_{\sigma}\right)$. We will prove that $\left\{\kappa \cdot \pi(\beta) \xi_{\iota} ; \iota \in I\right\}$ converges strongly to 0 , that is,

$$
\left\|\kappa \cdot \pi(\beta) \xi_{\iota}\right\|_{L^{2}\left(G_{0}, \mathscr{T}_{\sigma}\right)}^{2}=\int_{G_{0}}\left|\kappa\left(x_{0}\right)\right|^{2}\left\|\pi(\beta) \xi_{\iota}\left(x_{0}\right)\right\|_{\mathscr{H}_{\sigma}}^{2} d x_{0}
$$

converges to 0 .

From the assumption, $\left\{R\left(x_{0}\right) \xi_{c}\right\}_{c \in I}$ converges weakly to 0 in $\mathscr{H}_{\sigma}$ for each fixed $x_{0} \in G_{0}$. Since $\sigma^{x_{0}}$ is an irreducible unitary representation of the nilpotent group $N$, which is liminal (CCR), $\sigma^{x_{0}}\left(\beta_{N}\right)$ is a compact operator on $\mathscr{H}_{\sigma}$. Therefore, $\left\{\sigma^{x_{0}}\left(\beta_{N}\right) R\left(x_{0}\right) \xi_{\iota}\right\}_{\iota \in I}$ converges strongly to 0 in $\mathscr{H}_{\sigma}$, that is, $\left\|\left(\pi(\beta) \xi_{\iota}\right)\left(x_{0}\right)\right\|_{\mathscr{A}_{\sigma}}$ converges to 0 for each $x_{0} \in G_{0}$. To apply Lebesgue's dominated convergence theorem we now show that the integrand $\left|\kappa\left(x_{0}\right)\right|^{2}\left\|\left(\pi(\beta) \xi_{\iota}\right)\left(x_{0}\right)\right\|_{\mathscr{S}^{\sigma} \sigma}^{2}$ is uniformly bounded by an integrable function. Since $\left\{\xi_{\iota}\right\}_{\iota \in I}$ converges weakly, we have $\left\|\xi_{\iota}\right\|_{L^{2}\left(G_{0}, \mathscr{H}_{\sigma}\right)} \leqq C_{0}$ for some positive constant $C_{0}$. Then using the inequalities $\left\|\sigma^{x_{0}}\left(\beta_{N}\right)\right\| \leqq\left\|\beta_{N}\right\|_{L^{1(N)}}$ and $(*)$, we get

$$
\begin{aligned}
\left\|\left(\pi(\beta) \xi_{c}\right)\left(x_{0}\right)\right\|_{\mathscr{H}_{\sigma}} & =\left\|\sigma^{x_{0}}\left(\beta_{N}\right) R\left(x_{0}\right) \xi_{c}\right\|_{\mathscr{H}_{\sigma}} \\
& \leqq\left\|\sigma^{x_{0}}\left(\beta_{N}\right)\right\|\left\|R\left(x_{0}\right) \xi_{c}\right\|_{\mathscr{H}_{\sigma}} \\
& \leqq\left\|\left(\beta_{N}\right)\right\|_{\left.L^{1(N)}\right)} \Delta_{G_{0}}^{1 / 2}\left(x_{0}\right)\left\|\beta_{0} \cdot \Delta_{G}\right\|_{L^{2}\left(G_{0}\right)} C_{0},
\end{aligned}
$$

i.e., $\left\|\left(\pi(\beta) \xi_{\iota}\right)\left(x_{0}\right)\right\|_{\mathscr{A}_{\sigma}} \leqq C \Delta_{G_{0}}^{1 / 2}\left(x_{0}\right)$, where $C$ is a constant independent of $\iota$. Thus

$$
\left|\kappa\left(x_{0}\right)\right|^{2}\left\|\left(\pi(\beta) \xi_{c}\right)\left(x_{0}\right)\right\|_{\mathscr{H}_{\sigma}}^{2} \leqq C^{2}\left|\kappa\left(x_{0}\right)\right|^{2} \Delta_{G_{0}}\left(x_{0}\right),
$$

where the right hand side is a continuous function with compact support. This verifies that $\left\|\kappa \cdot \pi(\beta) \xi_{\iota}\right\|_{L^{2}\left(G_{0}, \mathscr{G}_{\sigma}\right)}$ converges to 0 , which proves the lemma. 
We now return to the proof of the theorem. Let $\varphi$ be an $L^{1}$-function satisfying the condition of claim 2 . In order to prove that $\pi(\varphi)$ is a compact operator, we show that it can be approximated (with the operator norm) by compact operators.

Let $\left\{K_{m}\right\}_{m \in N}$ be a family of compact sets in $G_{0}$ such that $K_{m} \subset \operatorname{int}\left(K_{m+1}\right)$ (the interior of $K_{m+1}$ ) and $\bigcup_{m \in N} K_{m}=G_{0}$, and $\kappa_{m} \in C_{c}\left(G_{0}\right)$ which satifies the following:

$$
\kappa_{m}(x)=\left\{\begin{array}{ll}
1, & x \in K_{m} \\
0, & x \notin K_{m+1}
\end{array}, \quad 0 \leqq \kappa_{m}(x) \leqq 1 \text { for all } x \in G_{0} .\right.
$$

Choose a sequence $\left\{\beta_{\nu}\right\}_{\nu \in N}$ of continuous functions on $G$ such that $\left\|\varphi-\beta_{\nu}\right\|_{L^{1}(G)} \rightarrow 0$ as $\nu \rightarrow \infty$ and $\beta_{\nu}=\sum_{c=1}^{M_{\nu}} \beta_{N}^{c} \cdot \beta_{0}^{c}, \quad \beta_{N}^{c} \in C_{c}(N), \beta_{0}^{c} \in C_{c}\left(G_{0}\right)$, for all $\nu$. For each $m$ and $\beta_{\nu}$ define a bounded operator $\pi_{m}\left(\beta_{\nu}\right)=\kappa_{m} \cdot \pi\left(\beta_{\nu}\right)=\sum_{\iota=1}^{M_{\nu}} \kappa_{m} \cdot \pi\left(\beta_{N}^{c} \cdot \beta_{0}^{c}\right)$, which is compact by Lemma 2.4. We will show that $\left\|\pi(\varphi)-\pi_{m}\left(\beta_{\nu}\right)\right\| \rightarrow 0$ as $m, \nu \rightarrow \infty$. Since:

$$
\begin{aligned}
\left\|\pi(\varphi)-\pi_{m}\left(\beta_{\nu}\right)\right\| & \leqq\left\|\pi(\varphi)-\pi\left(\beta_{\nu}\right)\right\|+\left\|\pi\left(\beta_{\nu}\right)-\pi_{m}\left(\beta_{\nu}\right)\right\| \\
& \leqq\left\|\varphi-\beta_{\nu}\right\|_{L^{1(G)}}+\left\|\left(1-\kappa_{m}\right) \cdot \pi\left(\beta_{\nu}\right)\right\|,
\end{aligned}
$$

it is sufficient to show that $\left\|\left(1-\kappa_{m}\right) \cdot \pi\left(\beta_{\nu}\right)\right\| \rightarrow 0$. For arbitrary elements $\xi, \eta$ of $L^{2}\left(G_{0}, \mathscr{H}_{\sigma}\right)$,

$$
\begin{aligned}
& \left|\left\langle\left(1-\kappa_{m}\right) \cdot \pi\left(\beta_{\nu}\right) \xi, \eta\right\rangle_{L^{2}\left(G_{0}, \mathscr{r}_{\sigma}\right)}\right| \\
& =\left|\int_{G} \int_{G_{0}}\left\langle\left(1-\kappa_{m}\right)\left(x_{0}\right)(\pi(g) \xi)\left(x_{0}\right), \eta\left(x_{0}\right)\right\rangle_{\mathscr{F}_{\sigma}} d x_{0} \beta_{\nu}(g) d g\right| \\
& =\mid \int_{g_{1}} \int_{R^{2} k} \int_{G_{0}} \int_{G_{0}}\left\langle\left(1-\kappa_{m}\right)\left(x_{0}\right) \chi_{x_{0}^{-1} \cdot f}(\exp X) \sigma^{x_{0}}(\Psi(\boldsymbol{y})) \xi\left(x_{0} g_{0}\right), \eta\left(x_{0}\right)\right\rangle_{\mathscr{F}_{\sigma}} \\
& \cdot d x_{0} \sum_{l=1}^{M_{\nu}} \beta_{N}^{c}((\exp X) \Psi(\boldsymbol{y})) \beta_{0}^{c}\left(g_{0}\right) \Delta_{G}\left(g_{0}\right) d X d \boldsymbol{y} d g_{0} \\
& =\mid \int_{R^{2 k}} \int_{G_{0}} \int_{G_{0}}\left(1-\kappa_{m}\right)\left(x_{0}\right)\left\langle\sigma^{x_{0}}(\Psi(\boldsymbol{y})) \xi\left(x_{0} g_{0}\right), \eta\left(x_{0}\right)\right\rangle_{\mathfrak{A}_{\sigma}} \\
& \cdot \sum_{c=1}^{M_{\nu}} \mathscr{F}_{1} \beta_{N}^{c}\left(x_{0}^{-1} \cdot f_{1}\right)(\Psi(\boldsymbol{y})) \beta_{0}^{c}\left(g_{0}\right) d x_{0} \Delta_{G}\left(g_{0}\right) d \boldsymbol{y} d g_{0} \\
& \leqq \int_{\boldsymbol{R}^{2 k}} \int_{G_{0}} \sup _{x_{0} \in G_{0}}\left|\left(1-\boldsymbol{\kappa}_{m}\right)\left(x_{0}\right) \sum_{c=1}^{M_{\mathcal{L}}} \Phi_{1} \beta_{N}^{c}\left(x_{0}^{-1} \cdot f_{1}\right)(\Psi(\boldsymbol{y})) \beta_{0}^{c}\left(g_{0}\right)\right| \\
& \cdot \int_{G_{0}}\left|\left\langle\boldsymbol{\sigma}^{x_{0}}(\Psi(\boldsymbol{y})) \xi\left(x_{0} g_{0}\right), \eta\left(x_{0}\right)\right\rangle_{\mathscr{H}_{\sigma}}\right| d x_{0} \Delta_{G}\left(g_{0}\right) d \boldsymbol{y} d g_{0} \\
& \leqq \int_{\boldsymbol{R}^{2} k} \int_{G_{0}} \sup _{x_{0} \in G_{0}}\left|\left(1-\kappa_{m}\right)\left(x_{0}\right) \sum_{\iota=1}^{M_{\nu}} \mathscr{I}_{1} \beta_{N}^{\iota}\left(x_{0}^{-1} \cdot f_{1}\right)(\Psi(\boldsymbol{y})) \beta_{0}^{c}\left(g_{0}\right)\right| \\
& \text { - } \Delta_{G}\left(g_{0}\right) d \boldsymbol{y} d g_{0}\|\boldsymbol{\xi}\|\|\eta\| \text {. }
\end{aligned}
$$

(For $\beta_{N}^{\prime} \in C_{c}(N), \quad \mathscr{F}_{1} \beta_{N}^{\prime}$ is defined similarly to Definition 2.1: $\mathscr{I}_{1} \beta_{N}^{\prime}(l)(n)=$ $\int_{\mathfrak{g}_{1}} e^{\mathcal{N}-\beta_{1}\langle l, X\rangle} \beta_{N}^{c}((\exp X) n) d X, l \in \mathfrak{g}_{1}^{*}, n \in N$.) Here we have 


$$
\begin{aligned}
& \sup _{x_{0} \in G_{0}}\left|\left(1-\kappa_{m}\right)\left(x_{0}\right) \sum_{l=1}^{M_{\nu}} \mathscr{F}_{1} \beta_{N}^{c}\left(x_{0}^{-1} \cdot f_{1}\right)(\Psi(\boldsymbol{y})) \beta_{0}^{c}\left(g_{0}\right)\right| \\
\leqq & \sup _{l \in\left(G_{0} \cdot f_{1} \backslash K_{m}^{-1} \cdot f_{1}\right)}\left|\sum_{l=1}^{M_{\nu}} \mathscr{F}_{1} \beta_{N}^{c}(l)(\Psi(\boldsymbol{y})) \beta_{0}^{c}\left(g_{0}\right)\right| \\
\leqq & \sup _{l \in\left(G_{0} \cdot f_{1} \backslash K_{m}^{-1} \cdot f_{1}\right)}\left|\sum_{l=1}^{M_{\nu}} \mathscr{F}_{1} \beta_{N}^{c}(l)(\Psi(\boldsymbol{y})) \beta_{0}^{c}\left(g_{0}\right)-\mathscr{F}_{1} \varphi(l)\left(\Psi(\boldsymbol{y}) g_{0}\right)\right| \\
& +\sup _{l \in\left(G_{0} \cdot f_{1} \backslash K_{m}^{-1} \cdot f_{1}\right)}\left|\mathscr{F}_{1} \varphi(l)\left(\Psi(y) g_{0}\right)\right| .
\end{aligned}
$$

Writing

$$
\gamma_{m}\left(\Psi(\boldsymbol{y}) g_{0}\right)=\sup _{l \in\left(G_{0} \cdot f_{1} \backslash K_{m}^{-1} \cdot f_{1}\right)}\left|\mathscr{F}_{1} \varphi(l)\left(\Psi(\boldsymbol{y}) g_{0}\right)\right|,
$$

for brevity, we have

$$
\begin{aligned}
& \quad\left\|\left(1-\kappa_{m}\right) \pi\left(\beta_{\nu}\right)\right\| \\
& \leqq \\
& \qquad \int_{R^{2 k}} \int_{G_{0}}\left\{\sup _{l \in\left(G_{0} \cdot f_{\left.1 \backslash K_{m}^{-1} \cdot f_{1}\right)}\right.}\left|\sum_{l=1}^{M_{\nu}} \mathscr{F}_{1} \beta_{N}^{c}(l)(\Psi(\boldsymbol{y})) \beta_{0}^{\prime}\left(g_{0}\right)-\mathscr{F}_{1} \varphi(l)\left(\Psi(\boldsymbol{y}) g_{0}\right)\right|\right. \\
& \left.\quad \quad+\gamma_{m}\left(\Psi(\boldsymbol{y}) g_{0}\right)\right\} \Delta_{G}\left(g_{0}\right) d \boldsymbol{y} d g_{0} \\
& \leqq
\end{aligned}
$$

To show that the second term converges to 0 , we assert that for almost all $\Psi(\boldsymbol{y}) g_{0}$,

$$
\gamma_{m}\left(\Psi(\boldsymbol{y}) g_{0}\right) \longrightarrow 0 \text { as } m \rightarrow \infty .
$$

In fact, for each fixed $\Psi(\boldsymbol{y}) g_{0}$, the sequence $\left\{\gamma_{m}\left(\Psi(\boldsymbol{y}) g_{0}\right)\right\}_{m \in N}$ converges as $m \rightarrow \infty$ since it is nonnegative and monotonically decreasing. Suppose it converges to $\varepsilon>0$. Then we can choose a sequence $\left\{l_{m}=l_{m}\left(\Psi(\boldsymbol{y}) g_{0}\right)\right\}_{m \in N}$ in $g_{1}^{*}$ such that $l_{m} \in\left(G_{0} \cdot f_{1} \backslash K_{m}^{-1} \cdot f_{1}\right)$ and $\left|\mathscr{F}_{1} \varphi\left(l_{m}\right)\left(\Psi(\boldsymbol{y}) g_{0}\right)\right| \geqq \varepsilon$ for all $m \in \boldsymbol{N}$. Since the function $l \rightarrow \mathscr{F}_{1}(l)\left(\Psi(\boldsymbol{y}) g_{0}\right)$ on $\boldsymbol{g}_{1}^{*}$ tends to 0 as $l \rightarrow \infty$ for almost all $\Psi(\boldsymbol{y}) g_{0}$, $\left\{l_{m}\right\}_{m \in N}$ is bounded, and choosing a subsequence, if necessary, we may assume that $\left\{l_{m}\right\}_{m \in N}$ converges to a point $l_{0} \in g_{1}^{*}$ as $m \rightarrow \infty$. Then $l_{0} \in \partial\left(G_{0} \cdot f_{1}\right)$. In fact, if $l_{0} \in G_{0} \cdot f_{1}$, there exists a number $m_{0}$ such that $l_{0} \in K_{m}^{-1} \cdot f_{1}$ for all $m>m_{0}$, which contradicts our definition of $l_{0}$. Noting that $l \rightarrow \mathscr{I}_{1} \varphi(l)\left(\Psi(\boldsymbol{y}) g_{0}\right)$ is continuous, we have

$$
\left|\mathscr{F}_{1} \varphi\left(l_{m}\right)\left(\Psi(\boldsymbol{y}) g_{0}\right)\right| \longrightarrow\left|\mathscr{F}_{1} \varphi\left(l_{0}\right)\left(\Psi(\boldsymbol{y}) g_{0}\right)\right| \geqq \varepsilon \text { as } m \rightarrow \infty
$$

for almost all $\Psi(\boldsymbol{y}) g_{0}$. This contradicts the condition of claim 2, and our assertion is verified.

From the inequality $\sup _{l}\left|\mathscr{F}_{1} \varphi(l)\left(\Psi(\boldsymbol{y}) g_{0}\right)\right| \leqq \int_{g_{1}}\left|\varphi\left((\exp X) \Psi(\boldsymbol{y}) g_{0}\right)\right| d X$, $\gamma_{m}\left(\Psi(\boldsymbol{y}) g_{0}\right) \Delta_{G}\left(g_{0}\right)$ is uniformly, bounded by the integrable function $\int_{\mathfrak{g}_{1}}\left|\varphi\left((\exp X) \Psi(\boldsymbol{y}) g_{0}\right)\right| d X \Delta_{G}\left(g_{0}\right)$ on $\boldsymbol{R}^{2 k} \times G_{0}$. Thus $\int_{R^{2 k}} \int_{g_{1}} \gamma_{m}\left(\Psi(\boldsymbol{y}) g_{0}\right) \Delta_{G}\left(g_{0}\right) d \boldsymbol{y} d g_{0}$ 
$\rightarrow 0$ as $m \rightarrow \infty$. Hence $\left\|\left(1-\kappa_{m}\right) \cdot \pi\left(\beta_{\nu}\right)\right\| \rightarrow 0$ as $m, \nu \rightarrow \infty$. This gives claim 1 , and the proof of Theorem 2.2 finishes.

Corollary 2.5. Let $G=\operatorname{expg}, \pi$ and $\Omega$ be as in Theorem 2.2. Then the kernel of the Fourier transform with respect to $\pi$ is

$$
\left\{\varphi \in L^{1}(G) ; \mathscr{I}_{1} \varphi\left(l_{1}\right)(g)=0 \quad \text { for all } l_{1}=\left.l\right|_{\mathfrak{g}_{1}} \text { such that } l \in \Omega\right\} .
$$

PROOF. The assertion is easily verified in a similar way to Step 1 of the proof of Theorem 2.2.

\section{Examples.}

We first refer to Khalil's result for the $a x+b$ group.

ExAmple 3.1 [5], [7]. Let $G$ be the $a x+b$ group (Example 1.2). We denote an element $g=\left(\begin{array}{ll}a & b \\ 0 & 1\end{array}\right)$ of $G$ by $(b, a)$, then $d g=a^{-2} d b d a$ is a left Haar measure of $G$. The coadjoint orbits are (1) a single point $\lambda X^{*}, \lambda \in \boldsymbol{R}$, (2) an open half plane $\Omega_{+}=\left\{\beta X^{*}+\gamma Y^{*} ; \gamma>0, \beta, \gamma \in \boldsymbol{R}\right\}, \Omega_{-}=\left\{\beta X^{*}+\gamma Y^{*} ; \gamma<0, \beta, \gamma \in \boldsymbol{R}\right\} . \quad$ Let $\pi_{+}, \pi_{-}$be representations corresponding to $\Omega_{+}, \Omega_{-}$respectively. They are all the infinite dimensional irreducible representations of $G$ (since orbits of (1) correspond to characters). Then for $\varphi \in L^{1}(G)$ the following conditions are equivalent.

(a) Both $\pi_{+}(\varphi)$ and $\pi_{-}(\varphi)$ are compact operators.

(b) $\int_{R} \varphi(b, a) d b=0$ for almost all $a \in \boldsymbol{R}_{+}^{*}$.

Remark that $\partial \Omega_{+}=\partial \Omega_{-}=\boldsymbol{R} X^{*}$ in this case, from which the above statements follow.

EXAMPLE 3.2. Let $\mathfrak{g}=\boldsymbol{R}$-span $\left\{X_{1}, X_{2}, W, Z, Y_{1}, Y_{2}\right\}$ be the Lie algebra of Example 1.3 and $G=\operatorname{expg}$. Then $g=g_{0}+g_{1}$ (semi-direct), where $g_{0}=\boldsymbol{R}$ $\operatorname{span}\left\{X_{1}, X_{2}, W\right\}, \mathrm{g}_{1}=\boldsymbol{R}$-span $\left\{Z, Y_{1}, Y_{2}\right\}$ (see 1.5). In this case, there are four open orbits $\Omega_{i}(i=1,2,3,4)$ described as follows: Defining the polynomials $P_{1}, P_{2}$ on $\mathrm{g}^{*}$ by

we have

$$
\begin{aligned}
& P_{1}(f)=f\left(Y_{1}\right) f\left(Y_{2}\right)-\frac{1}{2} f(Z)^{2}, \\
& P_{2}(f)=f\left(Y_{2}\right),
\end{aligned}
$$

$$
\begin{aligned}
& \Omega_{1}=\left\{l \in \mathrm{g}^{*} ; P_{1}(l)>0, P_{2}(l)>0\right\}, \\
& \Omega_{2}=\left\{l \in \mathrm{g}^{*} ; P_{1}(l)<0, P_{2}(l)>0\right\}, \\
& \Omega_{3}=\left\{l \in \mathrm{g}^{*} ; P_{1}(l)>0, P_{2}(l)<0\right\},
\end{aligned}
$$




$$
\Omega_{4}=\left\{l \in \mathrm{g}^{*} ; P_{1}(l)<0, P_{2}(l)<0\right\} .
$$

Let $p: g^{*} \rightarrow g_{1}^{*}$ denote the natural projection defined by restriction, and $\left\{Y_{1}^{*}, Y_{2}^{*}, Z^{*}\right\}$ be the dual basis of $\left\{Y_{1}, Y_{2}, Z\right\}$ (regarded as a basis of $\mathfrak{g}_{1}$ ). Then

$$
\begin{aligned}
& p\left(\partial \Omega_{1}\right)=\left\{l \in \mathrm{g}_{1}^{*} ; P_{1}(l)=0, P_{2}(l) \geqq 0\right\}, \quad \text { i. e., the cone obtained by } \\
& \quad r S_{+}(\theta)=r\left(\left(Y_{1}^{*}+Y_{2}^{*}\right)+\cos \theta\left(Y_{1}^{*}-Y_{2}^{*}\right)+\sqrt{2} \sin \theta Z^{*}\right) ; r \geqq 0,0 \leqq \theta<2 \pi . \\
& p\left(\partial \Omega_{2}\right)=p\left(\partial \Omega_{1}\right) \cup Y_{2}^{\perp}, \text { where } Y_{2}^{\perp}=\left\{l \in \mathrm{g}_{1}^{*} ; l\left(Y_{2}\right)=0\right\} . \\
& p\left(\partial \Omega_{3}\right)=\left\{l \in \mathrm{g}_{1}^{*} ; P_{1}(l)=0, P_{2}(l) \leqq 0\right\}, \quad \text { i. e., the cone obtained by } \\
& r S_{-}(\theta)=r\left(-\left(Y_{1}^{*}+Y_{2}^{*}\right)+\cos \theta\left(Y_{1}^{*}-Y_{2}^{*}\right)+\sqrt{2} \sin \theta Z^{*}\right) ; r \geqq 0,0 \leqq \theta<2 \pi . \\
& p\left(\partial \Omega_{4}\right)=p\left(\partial \Omega_{3}\right) \cup Y_{2}^{\perp} .
\end{aligned}
$$

According to the realization of $g$ in Example 1.3, we regard $G$ as follows:

$$
G=G_{1} G_{0}=G_{1} \exp \boldsymbol{R} W \exp \left(\boldsymbol{R} X_{1}+\boldsymbol{R} X_{2}\right)
$$

$$
=\left\{g=\left(y_{1}, y_{2}, z, w, x_{1}, x_{2}\right)=\left(\begin{array}{cccc}
e^{x_{2}} & w e^{\left(x_{1}+x_{2}\right) / 2} & \frac{1}{2} w^{2} e^{x_{1}} & y_{2} \\
0 & e^{\left(x_{1}+x_{2}\right) / 2} & w e^{x_{1}} & z \\
0 & 0 & e^{x_{1}} & y_{1} \\
0 & 0 & 0 & 1
\end{array}\right) ;\right.
$$

Then the modular function of $G$ is $\Delta_{G}(g)=e^{-x_{1}-2 x_{2}}$, and we use the left Haar measure $d g=e^{-x_{1}-2 x_{2}} d y_{1} d y_{2} d z d w d x_{1} d x_{2}$, where $d w d x_{1} d x_{2}$ is a right Haar measure on $G_{0}$.

Let $\pi_{i}$ denote the representation corresponding to $\Omega_{i}, 1 \leqq i \leqq 4$, and $\mathcal{S}\left(\pi_{i}\right)=$ $\left\{\varphi \in L^{1}(G) ; \pi_{i}(\varphi)\right.$ is a compact operator $\}$, then we have

$$
\begin{aligned}
\mathscr{I}\left(\pi_{1}\right)= & \left\{\varphi \in L^{1}(G) ; \mathscr{F}_{1} \varphi\left(r S_{+}(\theta)\right)\left(w, x_{1}, x_{2}\right)=0 \text { for all } r \geqq 0,0 \leqq \theta<2 \pi\right. \\
& \text { and } \left.d w d x_{1} d x_{2} \text {-almost all }\left(w, x_{1}, x_{2}\right)\right\}, \\
\mathcal{G}\left(\pi_{2}\right)= & \left\{\varphi \in L^{1}(G) ; \mathscr{I}_{1} \varphi\left(t Y_{1}^{*}+u Z^{*}\right)\left(w, x_{1}, x_{2}\right)=0, \mathscr{I}_{1} \varphi\left(r S_{+}(\theta)\right)\left(w, x_{1}, x_{2}\right)=0\right. \\
& \text { for all } \left.t, u \in \boldsymbol{R}, r \geqq 0,0 \leqq \theta<2 \pi \text { and almost all }\left(w, x_{1}, x_{2}\right)\right\}, \\
\mathcal{S}\left(\pi_{3}\right)= & \left\{\varphi \in L^{1}(G) ; \mathscr{I}_{1} \varphi\left(r S_{-}(\theta)\right)\left(w_{1}, x_{1}, x_{2}\right)=0 \text { for all } r \geqq 0,0 \leqq \theta<2 \pi\right. \\
& \text { and almost all } \left.\left(w, x_{1}, x_{2}\right)\right\}, \\
\mathcal{G}\left(\pi_{4}\right)= & \left\{\varphi \in L^{1}(G) ; \mathscr{F}_{1} \varphi\left(t Y_{1}^{*}+u Z^{*}\right)\left(w, x_{1}, x_{2}\right)=0, \mathscr{F}_{1} \varphi\left(r S_{-}(\theta)\right)\left(w, x_{1}, x_{2}\right)=0\right. \\
& \text { for all } \left.t, u \in \boldsymbol{R}, r \geqq 0,0 \leqq \theta<2 \pi \text { and almost all }\left(w, x_{1}, x_{2}\right)\right\},
\end{aligned}
$$

and

$$
\mathcal{S}\left(\pi_{1}\right) \supset \mathcal{G}\left(\pi_{2}\right), \mathcal{G}\left(\pi_{3}\right) \supset \mathcal{G}\left(\pi_{4}\right) .
$$


ACKNowledgements. I wish to express my sincere gratitude to Professor Hidénori Fujiwara for helpful discussions.

\section{References}

[1] G. Arsac, Opérateurs compacts dans l'espace d'une représentation, C.R. Acad. Sci. Paris, 286 (1978), 687-689.

[2] P. Bernat et al., Représentations des groupes de Lie résolubles, Dunod, Paris, 1972.

[3] I.K. Busyatskaya, Representations of exponential Lie groups, Functional Anal. Appl., 7 (1973), 151-152.

[4] M. Duflo et M. Rais, Sur l'analyse harmonique sur les groupes de Lie résolubles, Ann. Sci. École Norm. Sup., 9 (1976), 107-144.

[5] P. Eymard et M. Terp, La transformation de Fourier et son inverse sur le groupe des $a x+b$ d'un corps local, Lecture Notes in Math., 739, Springer, 1979, 207-248.

[6] S. Kaneyuki, Homogeneous bounded domains and Siegel domains, Lecture Notes in Math., 241, Springer, 1971.

[7] I. Khalil, Sur l'analyse harmonique du groupe affine de la droite, Studia Math., 51 (1974), 140-166.

[8] T. Nomura, Harmonic analysis on a nilpotent Lie group and representations of a solvable Lie group on $\bar{\partial}_{b}$ cohomology spaces, Japan. J. Math., 13 (1987), 277-332.

[9] L. Pukanszky, On unitary representations of exponential groups, J. Funct. Anal., 2 (1968), 73-113.

[10] I. I. Pyatetskii-Shapiro, Automorphic functions and the geometry of classical domains, Gordon and Breach, New York, 1969.

[11] H. Rossi, Lectures on representations of groups of holomorphic transformations of Siegel domains, Lecture Note, Brandeis University, 1972.

[12] H. Rossi et M. Vergne, Équations de Cauchy-Riemann tangentielles associées à un domaine de Siegel, Ann. Sci. École Norm. Sup., 9 (1976), 31-80.

Junko INOUE

Department of Mathematics

Faculty of Science

Kyushu University 33

Fukuoka 812

Japan 